\title{
Oral lichenoid lesions related to contact with dental materials: A literature review
}

\author{
María-José Cobos-Fuentes ${ }^{1}$, Ángel Martínez-Sahuquillo-Márquez ${ }^{2}$, Isabel Gallardo-Castillo ${ }^{3}$, Jose-Ramón \\ Armas-Padrón ${ }^{4}$, Ana Moreno-Fernández ${ }^{4}$, Pedro Bullón-Fernández ${ }^{5}$
}

\author{
${ }^{1}$ Associate Professor of Oral Medicine; Faculty of Dentistry; University of Seville \\ ${ }^{2}$ Full-time Professor of Oral Medicine; Faculty of Dentistry; University of Seville \\ ${ }^{3}$ Visiting Professor of Oral Medicine; Faculty of Dentistry; University of Seville \\ ${ }^{4}$ Full-time Professor of Pathological Anatomy; Department of Cell Biology and Normal or Pathological Histology; University \\ of Seville \\ ${ }^{5}$ Professor of Oral Medicine and Periodontology; University of Seville
}

Correspondence:

Facultad de Odontología

Universidad de Sevilla

Cl. Avicena, $s / n$

41009 Sevilla (Spain)

mjosecobos@gmail.com

Received: 21/11/2008

Accepted: 07/07/2009

Cobos-Fuentes MJ, Martínez-Sahuquillo-Márquez A, Gallardo-Castillo I, Armas-Padrón JR, Moreno-Fernández A, Bullón-Fernández P. Oral lichenoid lesions related to contact with dental materials: A literature review. Med Oral Patol Oral Cir Bucal. 2009 Oct 1;14 (10):e514-20. http://www.medicinaoral.com/medoralfree01/v14i10/medoralv14i10p514.pdf

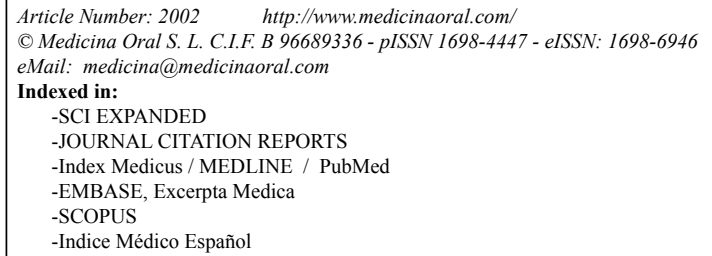

\begin{abstract}
Oral lichenoid lesions related to contact are defined as oral-cavity eruptions with an identifiable etiology, and are clinically and histologically similar to oral lichen planus. Within this group are found oral lichenoid lesions related to contact with dental materials (OLLC), the most common being those related to silver amalgam. Currently, it remains difficult to diagnose these lesions due to the clinical and histopathological similarity with oral lichen planus and other oral mucosa lesions of lichenoid characteristics. In the present paper, we carry out an updated review of the tests for, and the different characteristics of OLLC, which may aid the diagnosis. For this review, we made searches in the Pubmed ${ }^{\circledR}$ and Cochrane ${ }^{\circledR}$ databases. Among the literature we found several published papers, from which we have used review papers, case papers, cohort studies, case and control studies, and a meta-analysis study. After carrying out this review, we can conclude that the diagnosis of these lesions is still difficult and controversial. However, there are different aspects in the clinical presentation, pathological study and results obtained when replacing suspect materials, which, when taken together, may be useful when establishing the final diagnosis of OLLC.
\end{abstract}

Key words: Oral lichenoid lesion related to contact, dental materials, amalgam, patch test. 


\section{Introduction}

The term oral lichenoid lesion (OLL) is used to describe eruptions of the oral cavity having an identifiable etiology, which are clinically and histologically similar to oral lichen planus (OLP) (1). In the literature, different terms are used to refer to these lesions. OLLs have sometimes been considered as part of OLP $(2,3)$, and have also been described as contact allergies (4), oral lichenoid lesions $(5,6)$, contact lesions $(7)$, or oral lichenoid reactions (OLR) (8).

The term OLR was proposed by Finne in 1982 (2) to designate clinically indistinguishable lesions of OLP -in which a specific etiological factor (mercury in Finne's original description) can be inferred and/or demonstrated - and to differentiate this from the generically idiopathic OLP. Since this concept was proposed, these lesions have been described as a response to a wide variety of triggering factors and said to involve several clinical types. A. OLL related to contact (OLLC) as result of allergic contact-stomatitis (immune retarded hypersensitivity mediated by cells). These have been discovered to be in direct topographic relation with dental restorative materials, most commonly with amalgam. B. OLL related to drugs (OLLD) in which oral and/or skin lesions appear in temporal association with the ingestion of certain drugs, mainly oral hypoglycaemic drugs, inhibitors of the enzyme which transforms angiotensin and non-steroidal anti-inflammatory drugs. C. Oral lichenoid lesions in patients suffering from acute graftversus-host disease - more frequently in its chronic form - and lesions related to systemic diseases such as lupus erythematosus (9).

The objective of the present paper is to review the published literature related to the first group: lesions related to dental materials. For such review we carried out a search in July 2007 in the Pubmed $\AA$ and Cochrane ${ }^{\circledR}$ databases. The keywords used were: oral lichenoid lesions, oral lichenoid reactions, oral contact allergy and amalgam fillings AND contact allergy. The limits established were papers published in Spanish or English. We found different published papers, from which we selected review papers, case papers, cohort studies, case and control studies, and a meta-analysis study. With these articles we have attempted to deepen our knowledge on the tests and the different clinical and histopathological characteristics of OLLC which may ease its diagnosis, with the aim of being able to carry out a differential diagnosis with OLP and other lesions of oral mucosa with lichenoid characteristics, as well as the handling and treatment of patients with these lesions.

\section{Oral lichenoid lesions related to contact with dental material}

OLLCs can be seen in direct topographic relation to a causal agent. This reaction is often attributed to dental restorative materials, mercury amalgam being the most common (10). The contact of oral mucosa with certain dental restorative materials or their corrosive byproducts may induce a sensitivity response, resulting in immunologically mediated damage of the keratinocytes of the basal stratum of the epithelium (11).

The incidence of hypersensitivity to dental-use materials seems scarce, much less than cutaneous forms. The majority of allergic reactions to dental materials correspond to hypersensitivity reactions type IV, mediated by cells (T lymphocytes). The causes of such low incidence may be that saliva sweeps, dilutes and makes allergens disappear quickly; low mucosa keratinization, which makes hapten-combination more difficult to take place; its high vascularization, which eliminates the allergens of the area; and the high resistance of oral mucosa (12). Although there is no unique and specific medical profile of intraoral contact allergy, lichenoid reactions seem to be the most usual manifestation of contact sensitization in oral mucosa.

- OLL related to contact with non-metallic dental materials

OLLs related to non-metallic dental materials are notably less frequent than those to metallic materials. The relation existing between allergies to acrylic materials of the prosthesis base and the presence of denture stomatitis has been discussed. Since the studies carried out by Fisher in 1959, the theory that denture stomatitis may be due to an allergic phenomenon has been discarded, but would rather be due to local irritation and to the presence of Candidas, although allergic etiology cannot be discarded in any case (13).

Hypersensitivity reactions to dental components have also been described; with resin monomers being identified as a cause. Reactions to HEMA, Bis-GMA and methacrylate resins - which are present in composites have also been described although it is rather infrequent to find free molecules after material light curing (14). Hypersensitivity reactions to composites are infrequent since free-monomer rates are lower than $1 \%$ of the whole product, being lower in materials of dual polymerization and higher in self-curing ones.

- OLL related to contact to silver amalgam

Although mercury amalgams have been the most-used restorative material in dentistry, cases of hypersensitivity to this material are relatively infrequent, the most common form of reaction being constituted by OLLs. These lesions are produced by hypersensitivity to one of its constituents, almost always mercury, but occasionally to others such as copper, zinc or tin (15).

Many oral mucosa lesions with lichenoid characteristics seem to be related to amalgam contact (11). Currently, the diagnosis of an OLL related to dental materials, according to Al-Hashimi et al. (9), depends fundamentally on: 1) Its clinical presentation; 2) Histological results; 3) 
Results of the patch test for mercury; and 4) Results of replacing suspect material $(5,8)$ :

1) Clinical presentation

The most common finding is OLLs closely related to an amalgam filling (complete or partial direct contact) (Figure 1).

According to the different publications, it is much more infrequent to find OLL in an oral cavity with amalgam fillings without direct contact, and which may be explained by excessive levels of total mercury in saliva. Even less common are distant or extraoral lesions, whose manifestations may occur in any area of the skin (being especially frequent in face and limbs) and especially in mucosa (16).

Oral mucosa OLLs related to amalgam may have variable manifestations such as stretch marks and plaques, or as erythematous, erosive, atrophic or ulcerative lesions. Buccal mucosa and the lateral edge of the tongue (Figure 2) are the most frequent locations, but in some cases the gingiva, lips and floor of the mouth can be affected. The clinical manifestations of OLLs are similar to those

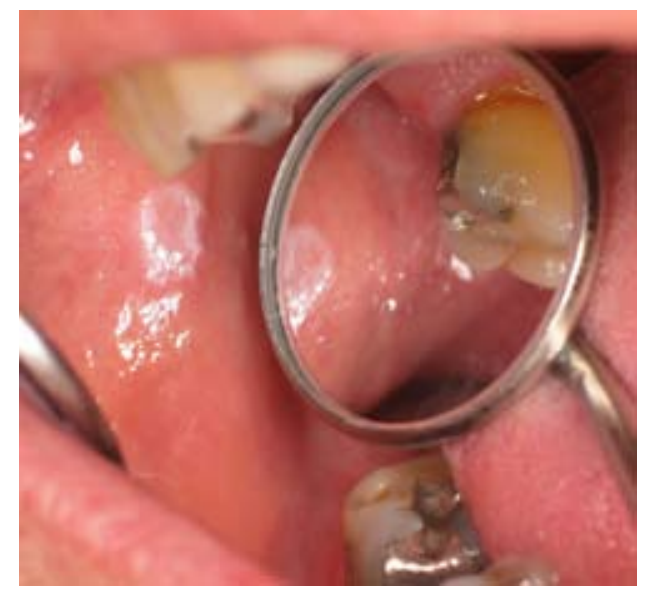

Fig. 1. OLL closely related to an amalgam filling.

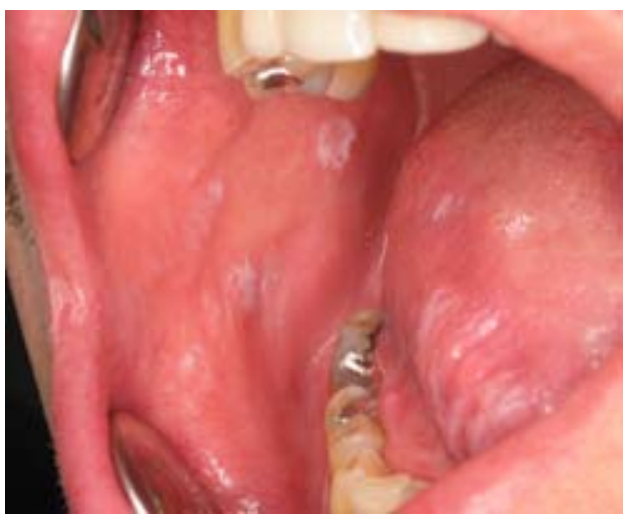

Fig. 2. OLLs located in buccal mucosa and the lateral edge of the tongue related to amalgam fillings. of OLP, although several authors such as Henriksson et al. (8) suggest that the medical profile of OLLs related to amalgam fillings differs from the classical clinical manifestations of OLP. Unlike OLP, OLLs caused by hypersensitivity are not usually symmetric and are in close vicinity to dental fillings or other contact agents $(15,17)$. In OLP, lesions may affect other locations of the oral mucosa unrelated to fillings such as gums and other mucocutaneous locations. The duration of the contact between oral mucosa and the causative material is an important factor for the development of these lesions (10).

Pain is the most prevalent symptom of OLLs and is generally related to atrophic or erosive forms. The range of patient symptoms oscillates from slight to severe, being moderate in most cases (8). Furthermore, several local oral complications can be observed such as metallic taste or dry mouth (18), which are difficult to relate to an allergic response. The prevalence of OLLs among women is approximately three times higher than in men, the highest range being found from the age of 50 onwards.

2) Histological results

It is not possible to confirm the diagnosis of OLLC through histology, due to the difficulty in differentiating OLP from OLLs from a histological viewpoint (10). Histopathologically, OLLs are characterized by a lichenoid tissue reaction in which two key findings can be found: A band-like lymphocytic infiltrate in lamina propria and a liquefactive degeneration of basal keratinocytes (9). According to the diagnostic criteria proposed by van der Meij et al., these lesions do not fulfil all clinical, and histopathological diagnostic criteria of an OLP typical lesion, and will be therefore diagnosed as OLL or compatible to OLP (19).

The study carried out by Thornhill et al. (11) confirms the difficulties in distinguishing OLLCs from OLP based only on histological findings. This difficulty can be due the activity of the disease, which varies over time, and so a wide spectrum of histopathological appearances can be observed in both OLLCs and OLP. They propose four histopathological findings useful for distinguishing between these two entities: an inflammatory infiltrate deeply located in some or all areas; a focal perivascular infiltrate; and the presence of plasmatic cells and neutrophils in the connective tissue.

3) Patch test results

The most-used lab test for contact allergy is the skin or patch test. Generally, diagnostic tests for intraoral allergy are carried out with epicutaneous patches. This is due to the extensive experience of allergists in handling and interpreting tests with epicutaneous patches, which are simple to carry out, require standard and simple material, and are cheap and relatively comfortable for both doctor and patient.

Epimucosa tests have been described with the aim of 
transferring the conditions of epicutaneous tests to oral mucosa. Several authors state that it is not necessary to carry out an Epimucosa test to detect contact allergies in oral mucosa, since the epicutaneous test provides appropriate information. Besides, skin tests are preferable, since their sensitivity and specificity are higher and the procedure is simpler. The most commonly used test is the epicutaneous test set of the dental series (Trolab $®$ ). The test materials are applied on normal skin on the back and can be interpreted after 72 hours of exposure. Patients are considered positive to an allergen if an erythematous, edematous (vesicular) or ulcerative reaction appears at the site of contact (3).

Currently, the use of epicutaneous patch tests to detect sensitivity to specific substances in patients suspected of suffering contact allergy is controversial. The result of the patch test may vary substantially depending on the components used, material concentration, vehicles used in the test, and test-evaluation criteria (20).

Several studies highlight the importance of testing a wide range of mercury allergens. In their study, Laine et al. conclude that the allergen test should include metallic mercury, $1 \%$ ammoniated mercury and $0.1 \%$ mercury chloride with the aim of detecting all cases of allergy (5).

A recent revision published by Al-Hashimi (9) on these lesions mentions that the application of the skin patch test to the suspect material -and to any other material which may be used as a substitute (gold, composite, glass ionomer cements and acrylic) - may be useful in certain cases, and that interpretations should be collected on the $3^{\text {rd }}, 7^{\text {th }}$ and $14^{\text {th }}$ days, or even later in order to avoid delayed reactions. The patch test may be useful for clinicians, mainly to determine which material would be convenient to replace the lesion-causing material (i.e., that to which the patient shows no reaction).

4) Results of replacing the suspect material

Treatment of oral lichenoid lesions related to contact consists of the removal, replacement or recovering of fillings in direct physical contact with mucosa lesions and are suspected of playing a causal role (9). The results of several clinical studies have shown that replacing silver-amalgam fillings with fillings made of other materials causes these OLLs to disappear or considerably improve within days or weeks $(4,5,7)$.

Figures for clinical recovery after replacement oscillate between 48 and 95\% (14). This means there are cases in which lesion recovery does not occur after eliminating the possible cause, and leads us to consider that either this is not really a purely allergic phenomenon or that diagnostic reliability is inappropriate; or a combination of both (21). None of the published studies have checked if lesions are reproduced after re-exposure to the causative agent.

A wide variety of criteria have been used to select pa- tients for amalgam filling replacement, such as clinical diagnosis, form of lesion, topographic relation between lesions and amalgam fillings, and patch test results. As substitution materials, gold, composite, porcelain, glass ionomer and acrylic have been used.

Several authors state that partial removal of amalgam fillings, i.e., only those in contact with OLLs, may be suffice to achieve proper recovery $(8,22)$. However, for patients with extensive atrophic and erosive lesions without amalgam contact, total removal is suggested as treatment (8). The percentage of patients who completely recover oscillates between $37.5 \%$ (7) and 100\% (23). The most noticeable recoveries are observed when there is direct contact between lesion and restoration, and the least noticeable when there is no such contact. But even when lesions are not in direct contact with the amalgam, patients experience recovery. This could be due to parafunctional aspects which may connect lesions and amalgam fillings (8).

On certain occasions, it can be observed that, in mouths with numerous amalgam fillings, and with only one contact-related OLL, when that filling is removed and the others remain, clinical manifestations disappear $(15,16$, $22,24)$. It is also notable how lesions disappear when the amalgam is not removed, but covered with a crown (15).

Replacing amalgams with other metallic alloys, particularly gold-based alloys, may hinder recovery from OLLs. According to several authors, gold might be immunologically linked to mercury. Furthermore, negative reactions to other antigenic metals in the patch test do not prevent future allergic sensitization to such metals. It is possible that the exposure of the subjects to other alloys (with noble and non-noble metals) may allow crossreactions among metals, resulting in an inappropriate solution for the treatment of OLLs (25).

Several previous studies suggest that the patch test is not useful in determining which patients may benefit from the replacement of amalgam fillings (7). On the other hand, we found some other studies (summarised in Table 1) in which positive results were obtained when fillings were replaced according to the results obtained in the patch test. Koch \& Bahmer (26) suggest that amalgam fillings should only be replaced in patients with positive patch tests and clinically objective lesions. These authors found a statistically significant difference in the effect of amalgam replacement between patients with positive and negative patch tests. Supporting this theory, Laine et al. (5) divided their patients with OLL into two groups: type I and type II. In type I, lesions were restricted to amalgam-contact areas. In type II, lesions exceeded amalgam-contact area. They found that $78.8 \%$ of patients with type-I lesions had a positive test to mercury and 44 (out of 47) of these patients either completely recovered or considerably improved after 


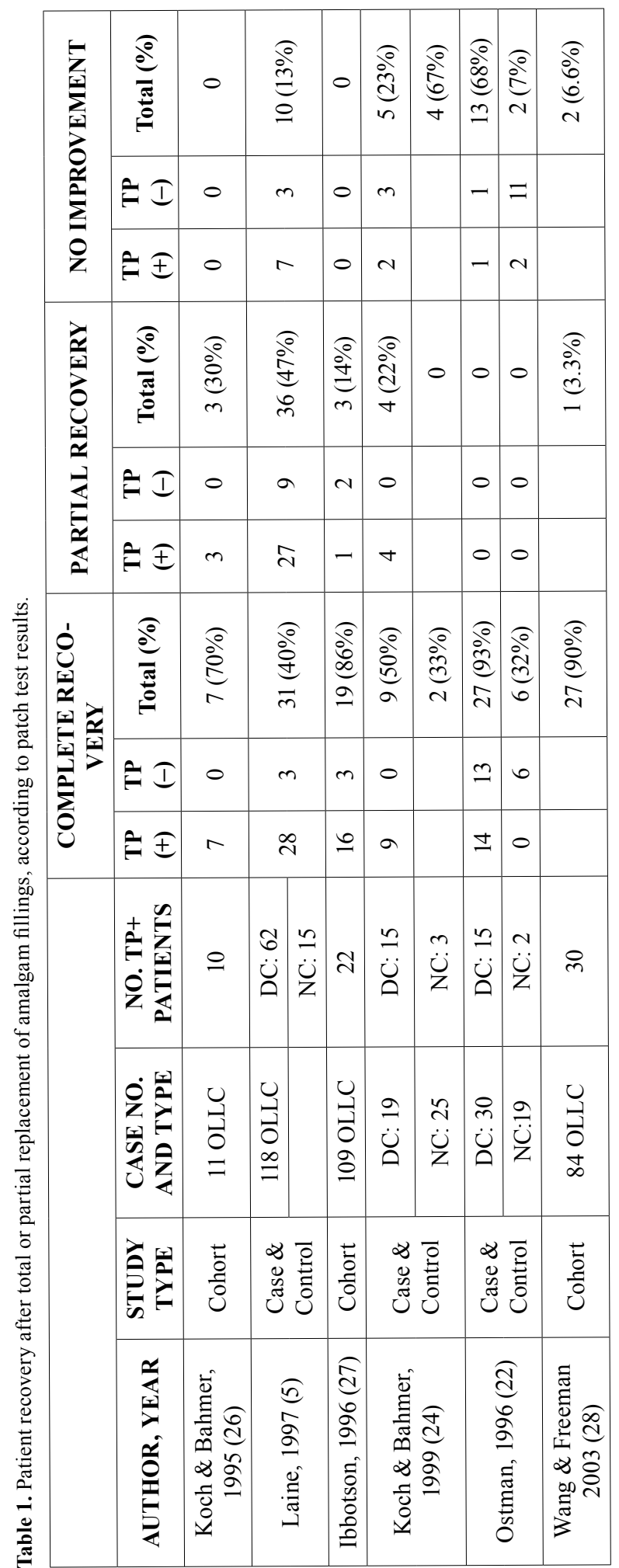

TP (+): Positive patch test; TP (-): Negative patch test; OLLC: Oral lichenoid lesions adjacent to amalgam fillings; DC: Lesions restricted to area of direct contact with amalgam fillings; NC: Lesions exceeding the area of contact with amalgam fillings replacing amalgam fillings, frequently within a period of 2-4 weeks.

Ibbotson et al. (27) observed that amalgam replacement allowed lesion resolution in 16 out of 17 patients with positive tests to mercury and in 3 out of 5 patients with negative results. However, this group carried out only one test interpretation at 72 hours. It has been observed that positive test interpretations may be delayed. Thus, Koch \& Bahmer (24) found in their study that 5 out of 15 patients with OLL in direct contact to amalgams only had positive tests to mercury 10 days after the test (3 patients) and 17 days after the test ( 2 patients).

Ostman et al. (22) conclude in their study that replacing amalgam fillings is more effective in lesions with direct contact than in those that extend beyond the filling contact area. However, this study establishes that there is no statistically significant difference in recovery between patients showing positive patch tests to mercury and those showing negative ones.

Wong \& Freeman (28) carried out a study with 84 patients with OLL adjacent to amalgam fillings with the aim of determining if the patch test is useful in identifying those patients who may respond favourably to amalgam filling removal. In 33 patients (39\%) the patch test was positive. Thirty out of 33 patients with positive patch tests to mercury had their amalgam fillings replaced by composite, gold or porcelain. Twenty-eight patients (87\%) noticed improvements in symptoms after three months. Their study determined that amalgam replacement is suitable in patients with positive patch tests to mercury. The data showed that patients with OLL and negative patch tests to mercury are asymptomatic or show much fewer symptoms than those with positive patch tests.

Issa et al. (20) carried out a literature review between 1966 and 2000 regarding OLL recovery after replacing amalgam fillings. Nineteen studies of variable quality were found: 14 cohort studies, and 5 case control studies. The studies collected treatments of 1,158 patients. Positive results in the patch test were obtained in $16 \%$ to $91 \%$ of these patients for at least one component of mercury. Six hundred and thirty-six out of the 1,158 patients replaced their fillings as a treatment measure. The monitoring period oscillated between 2 months and nine and a half years. The complete recovery range oscillated between $37.5 \%$ and $100 \%$. Improvement occurred within a short time (from one week to three months) of amalgam filling replacement. The greatest improvements were found in lesions in direct contact to amalgams. Observational studies suggest that the patch test is of limited value as an indicator for amalgam filling replacement and in predicting the results that will be obtained. The topographic relation between OLLs and amalgam fillings is a useful marker for prognosis, but it is not a determining indicator of the result. 
Table 2. Diagnostic criteria for oral lichenoid lesions related to contact.

\begin{tabular}{|l|l|}
\hline \multicolumn{1}{|c|}{ DIAGNOSTIC FINDINGS } & \multicolumn{1}{c|}{ COMMENTS } \\
\hline 1. Clinical presentation & $\begin{array}{l}\text { Asymmetric lesions and those in non-typical locations (15, } \\
17) \\
\text { Direct topographic relation between lesion and causing } \\
\text { material }(9,15,17)\end{array}$ \\
\hline 2. Histopathological findings & Compatible with OLP/OLL (19) \\
\hline 3. Patch test & $\begin{array}{l}\text { The patch test may help to determine the alternative mate- } \\
\text { rial which should be used }\end{array}$ \\
\hline $\begin{array}{l}\text { 4. Results of covering or replacing } \\
\text { the suspect restorative material }\end{array}$ & $\begin{array}{l}\text { Covering or replacing the suspicious and causing restor- } \\
\text { ative material may entail the disappearance of the lesion, } \\
\text { establishing its diagnosis (9) }\end{array}$ \\
\hline
\end{tabular}

It has been observed that there is only partial correspondence between positive patch test results and OLL recovery after amalgam replacement. While $90 \%$ of patients with positive patch test results improve after amalgam-filling replacement, only $68 \%$ do so when such test results have been negative. In spite of the high range of recovery in patients with positive patch test results, a significant percentage of patients with negative results also improve, which makes us consider the limitations of such a test as an indicator for amalgam filling removal (20).

In the studies by Thornhill et al. (11), the combination of a positive patch test and a strong clinical association between lesions and fillings was an excellent predictor of lesion recovery after amalgam replacement, and was a better predictor than either the patch test or clinical association alone.

After carrying out this review of the different diagnostic criteria proposed for OLLCs, we can conclude that currently the diagnosis of these lesions is still difficult and controversial. We consider that the criteria proposed by Al-Hashimi et al. (9) may help in the diagnosis of OLLC with regard to its clinical presentation, patch test results and treatment. However, these authors consider that the histopathological study is not always necessary, except when their clinical presentation is atypical in order to exclude the existence of malignancy. On this point, we consider that the histopathological study is always necessary, not only for discarding malignancy, but also because these lesions do not usually fulfill all the characteristic histopathological criteria of OLP and are diagnosed as compatible with OLP or OLL, according to the criteria proposed by van der Meij et al. (19), which can be very useful, together with the rest of findings (summarized in Table 2), to establish the final diagnosis of OLLCs.

\section{References}

1. Eisen D, Carrozzo M, Bagan Sebastian JV, Thongprasom K. Number V Oral lichen planus: clinical features and management. Oral Dis. 2005;11:338-49.

2. Finne K, Göransson K, Winckler L. Oral lichen planus and contact allergy to mercury. Int J Oral Surg. 1982;11:236-9.

3. Ismail SB, Kumar SK, Zain RB. Oral lichen planus and lichenoid reactions: etiopathogenesis, diagnosis, management and malignant transformation. J Oral Sci. 2007;49:89-106.

4. Bolewska J, Hansen HJ, Holmstrup P, Pindborg JJ, Stangerup M. Oral mucosal lesions related to silver amalgam restorations. Oral Surg Oral Med Oral Pathol. 1990;70:55-8.

5. Laine J, Kalimo K, Happonen RP. Contact allergy to dental restorative materials in patients with oral lichenoid lesions. Contact Dermatitis. 1997;36:141-6.

6. Pang BK, Freeman S. Oral lichenoid lesions caused by allergy to mercury in amalgam fillings. Contact Dermatitis. 1995;33:423-7.

7. Skoglund A. Value of epicutaneous patch testing in patients with oral, mucosal lesions of lichenoid character. Scand J Dent Res. 1994;102:216-22.

8. Henriksson E, Mattsson U, Håkansson J. Healing of lichenoid reactions following removal of amalgam. A clinical follow-up. J Clin Periodontol. 1995;22:287-94.

9. Al-Hashimi I, Schifter M, Lockhart PB, Wray D, Brennan M, Mig1iorati CA, et al. Oral lichen planus and oral lichenoid lesions: diagnostic and therapeutic considerations. Oral Surg Oral Med Oral Pathol Oral Radiol Endod. 2007;103 Suppl:S25.e1-12.

10. Thornhill MH, Sankar V, Xu XJ, Barrett AW, High AS, Odell EW, et al. The role of histopathological characteristics in distinguishing amalgam-associated oral lichenoid reactions and oral lichen planus. J Oral Pathol Med. 2006;35:233-40.

11. Thornhill MH, Pemberton MN, Simmons RK, Theaker ED. Amalgam-contact hypersensitivity lesions and oral lichen planus. Oral Surg Oral Med Oral Pathol Oral Radiol Endod. 2003;95:291-9.

12. Mallo Pérez L, Díaz Donado C. Intraoral contact allergy to materials used in dental practice. A critical review. Med Oral. 2003;8:33447.

13. Cumming CG, Wight C, Blackwell CL, Wray D. Denture stomatitis in the elderly. Oral Microbiol Immunol. 1990;5:82-5.

14. Kanerva L, Estlander T, Jolanki R. Allergic contact dermatitis from dental composite resins due to aromatic epoxy acrylates and aliphatic acrylates. Contact Dermatitis. 1989;20:201-11.

15. McGivern B, Pemberton M, Theaker ED, Buchanan JA, Thornhill $\mathrm{MH}$. Delayed and immediate hypersensitivity reactions associated with the use of amalgam. Br Dent J. 2000;188:73-6.

16. Yiannias JA, El-Azhary RA, Hand JH, Pakzad SY, Rogers RS 3rd. Relevant contact sensitivities in patients with the diagnosis of oral lichen planus. J Am Acad Dermatol. 2000;42:177-82. 
17. Pecegueiro M, Sachse MF, Amaro J, Farinha P, Fonseca I. Oral lichen planus versus oral lichenoid eruption as a manifestation of contact allergy. Contact Dermatitis. 1999;40:333-4.

18. Holmstrup P. Oral mucosa and skin reactions related to amalgam. Adv Dent Res. 1992;6:120-4.

19. van der Meij EH, Van der Waal I. Lack of clinicopathologic correlation in the diagnosis of oral lichen planus based on the presently available diagnostic criteria and suggestions for modifications. J Oral Pathol Med. 2003;32:507-12.

20. Issa Y, Brunton PA, Glenny AM, Duxbury AJ. Healing of oral lichenoid lesions after replacing amalgam restorations: a systematic review. Oral Surg Oral Med Oral Pathol Oral Radiol Endod. 2004;98:553-65.

21. De Rossi SS, Greenberg MS. Intraoral contact allergy: a literature review and case reports. J Am Dent Assoc. 1998;129:1435-41.

22. Ostman PO, Anneroth G, Skoglund A. Amalgam-associated oral lichenoid reactions. Clinical and histologic changes after removal of amalgam fillings. Oral Surg Oral Med Oral Pathol Oral Radiol Endod. 1996;81:459-65.

23. Alanko K, Kanerva L, Jolanki R, Kannas L, Estlander T. Oral mucosal diseases investigated by patch testing with a dental screening series. Contact Dermatitis. 1996;34:263-7.

24. Koch P, Bahmer FA. Oral lesions and symptoms related to metals used in dental restorations: a clinical, allergological, and histologic study. J Am Acad Dermatol. 1999;41:422-30.

25. Pigatto PD, Guzzi G. Oral lichenoid lesions: more than mercury. Oral Surg Oral Med Oral Pathol Oral Radiol Endod. 2005;100:398400

26. Koch P, Bahmer FA. Oral lichenoid lesions, mercury hypersensitivity and combined hypersensitivity to mercury and other metals: histologically-proven reproduction of the reaction by patch testing with metal salts. Contact Dermatitis. 1995;33:323-8.

27. Ibbotson SH, Speight EL, Macleod RI, Smart ER, Lawrence CM. The relevance and effect of amalgam replacement in subjects with oral lichenoid reactions. Br J Dermatol. 1996;134:420-3.

28. Wong L, Freeman S. Oral lichenoid lesions (OLL) and mercury in amalgam fillings. Contact Dermatitis. 2003;48:74-9. 